\title{
Two-Parameter Regularization Method for a Nonlinear Backward Heat Problem with a Conformable Derivative
}

\author{
Vu Ho $\left(D^{1},{ }^{1}\right.$ Donal O’Regan, ${ }^{2}$ and Hoa Ngo Van $\mathbb{D}^{2,3}$ \\ ${ }^{1}$ Faculty of Mathematical Economics, Banking University of Ho Chi Minh City, Ho Chi Minh City, Vietnam \\ ${ }^{2}$ Division of Computational Mathematics and Engineering, Institute for Computational Science, Ton Duc Thang University, \\ Ho Chi Minh City, Vietnam \\ ${ }^{3}$ Faculty of Mathematics and Statistics, Ton Duc Thang University, Ho Chi Minh City, Vietnam \\ Correspondence should be addressed to Hoa Ngo Van; ngovanhoa@tdtu.edu.vn
}

Received 19 November 2019; Revised 17 February 2020; Accepted 8 April 2020; Published 5 May 2020

Academic Editor: Honglei Xu

Copyright (c) $2020 \mathrm{Vu}$ Ho et al. This is an open access article distributed under the Creative Commons Attribution License, which permits unrestricted use, distribution, and reproduction in any medium, provided the original work is properly cited.

In this paper, we consider the nonlinear inverse-time heat problem with a conformable derivative concerning the time variable. This problem is severely ill posed. A new method on the modified integral equation based on two regularization parameters is proposed to regularize this problem. Numerical results are presented to illustrate the efficiency of the proposed method.

\section{Introduction}

Partial differential equations (PDEs) with different types of boundary conditions play an essential tool in modelling natural phenomena. For time-dependent phenomena, one usually adds an initial time condition or a final time condition, which can be considered as the data. For the timeinverse problem from the final data, the main goal is to reconstruct the whole structure in previous times. These problems were widely studied in the papers by Tikhonov and Arsenin [1], Glasko [2], and the references cited therein. An example is the backward heat problem (BHP) where the goal is to recover the previous status of a physical field from the present information. It is well known that the BHP is a classical ill-posed problem, and it is quite difficult to consider since the solution does not always exist. Furthermore, even if the solution does exist, the continuous dependence of the solution on the data is not guaranteed. The BHP has been considered in the literature using different methods (see [3-10] and the references cited therein). Fu et al. [3] applied a wavelet dual least square method to investigate a BHP with constant coefficients, in [4], Hao et al. gave an approximation for this problem using a nonlocal boundary value problem method, Hao and Duc [5] used the Tikhonov regularization method to give an approximation for this problem in a
Banach space, and Tautenhahn in [6] established an optimal error estimate for a backward heat equation with constant coefficients. Using the stabilized quasireversibility method, the final value problem for a class of nonlinear parabolic equations is investigated by Trong and Tuan [7], and in [8], the authors used the integral equation method to regularize the backward heat conduction problem and they obtained some error estimates. Tuan and Ngo [10] introduced the truncation method for solving the BHP and presented new error estimates for investigating the stability of the given problem. Also, the modified integral equation method and the modified quasiboundary value are extended to investigate inverse-time problems for axisymmetric backward heat equations in $[11,12]$ and the nonlinear spherically symmetric backward heat equation in [13].

The concept of the so-called conformable derivative was proposed by Khalil et al. [14] and discussed by Atangana et al. [15] and Abdeljawad [16]. Anderson and Ulness in [17] provided a potential application of the conformable derivative in quantum mechanics, Hammad and Khalil [18] used a conformable fourier series to interpret the solution of the conformable heat equation, and Chung [19] employed the conformable derivative concept to investigate the problem of Newtonian mechanics, and the Euler-Lagrange equation was also constructed. Eslami [20] employed the Kudryashov 
method to obtain the traveling wave solutions to the coupled nonlinear Schrodinger equation with a conformable derivative, Çenesiz et al. [21, 22] studied Burgers' equation, the modified Burgers's equation, and the Burgers-Korteweg-de Vries equation with a conformable derivative version, Çenesiz et al. [23] investigated the stochastic solution of conformable Cauchy problems where the space operators may correspond to Brownian motion or a Levy process, and $\mathrm{Vu}$ et al. [24] employed the quasiboundary value method to regularize the inverse-time problem for the nonhomogeneous heat equation with a conformable derivative, and a Hölder-type estimation error for the whole time interval was obtained. In this paper, we consider the following backward heat equations:

$$
\begin{aligned}
D_{t}^{\alpha} u(x, t)-u_{x x}(x, t) & =f(x, t), \quad(x, t) \in \Omega \times(0, T], \\
u(x, t) & =0, \quad(x, t) \in \partial \Omega \times(0, T], \\
u(x, T) & =g(x), \quad x \in \Omega,
\end{aligned}
$$

where $\Omega=[0, a], T$ is a positive number, the functions $f(x, t)$ and $g(x)$ are given, and $D_{t}^{\alpha}$ is the conformable derivative of order $\alpha$ with respect to $t$ defined by

$$
\begin{aligned}
& D_{t}^{\alpha} u(t)=\lim _{h \longrightarrow 0} \frac{u\left(t+h t^{1-\alpha}\right)-u(t)}{h}, \\
& D_{t}^{\alpha} u(0)=\lim _{t \rightarrow 0^{+}} D_{t}^{\alpha} u(t) .
\end{aligned}
$$

From the information given at final time $t=T$, the goal of the problem is to recover the information $u(x, t)$ for
$0 \leq t<T$. Unfortunately, BHP (1)-(3) is ill posed in the sense of Hadamard, i.e., it violates at least one of the following conditions:

(1) Existence. There exists a solution of the problem.

(2) Uniqueness. The solution must be unique.

(3) Stability. The solution must depend continuously on the data, i.e., any small error in given data must lead to a corresponding small error in the solution.

Using Theorem 1 in Section 2, we see that the solution of problems (1)-(3) is given by

$u(x, t)$

$$
=\sum_{n=1}^{\infty}\left(e^{k_{n}\left(b^{\alpha}-t^{\alpha} / \alpha\right)} g_{n}-\int_{t}^{b} s^{\alpha-1} e^{k_{n}\left(s^{\alpha}-t^{\alpha} / \alpha\right)} f_{n}(u)(s) \mathrm{d} s\right) \sin \left(\frac{n \pi}{a} x\right)
$$

where the terms in the above equation are given in Theorem 1. We observe that $\exp \left(k_{n}\left(b^{\alpha}-t^{\alpha} / \alpha\right)\right) \longrightarrow^{n} \longrightarrow \infty \infty$, so this yields an instability of the solution of problems (1)-(3). This violates condition (3), so problems (1)-(3) are ill posed. In this paper, to stabilize problems (1)-(3), we shall apply the modified integral equation method via a two-parameter regularization to regularize problems (1)-(3). To do this, we shall replace the above instability term by the term $\left(\varepsilon k_{n}+e^{-k_{n}\left(\left(b^{\alpha}+\gamma\right) / \alpha\right)}\right)^{\left(\left(t^{\alpha}-b^{\alpha}\right) /\left(b^{\alpha}+\gamma\right)\right)}$, where $t \in[0, b]$, $\varepsilon \in\left(0,\left(b^{\alpha}+\gamma\right) / \alpha\right), \alpha \in(0,1)$ is fixed, and $\gamma$ is a positive constant. From the proposed term, we use the following modified integral equation to approximate or to regularize the solution of problems (1)-(3):

$$
u^{\varepsilon, \gamma}(x, t)=\sum_{n=1}^{\infty}\left(\varepsilon k_{n}+e^{-k_{n}\left(\left(b^{\alpha}+\gamma\right) / \alpha\right)}\right)^{\left(\left(t^{\alpha}-b^{\alpha}\right) /\left(b^{\alpha}+\gamma\right)\right)}\left(g_{n}-\int_{t}^{b} s^{\alpha-1} e^{k_{n}\left(\left(s^{\alpha}-b^{\alpha}\right) / \alpha\right)} f_{n}\left(u^{\varepsilon, \gamma}\right)(s) \mathrm{d} s\right) \sin \left(\frac{n \pi x}{a}\right) .
$$

In Section 2, we show that problems (1)-(3) can be transformed into an integral equation (5). In Section 3.1, we prove that the regularized problem (6) is well posed in the sense of Hadamard in two cases, namely, $\alpha \in(1 / 2,1)$ and $\alpha \in(0,1)$. In Section 3.2, the error estimates between the regularized solution of problem (6) and the solution of problems (1)-(3) with the prior condition on the solution in two cases of exact data and nonexact data are presented. In particular, we show that

$$
\begin{aligned}
& \left\|u^{\varepsilon, \gamma}(x, t)-u(x, t)\right\| \\
& \quad \leq \mathbb{D} \varepsilon^{\left(t^{\alpha}+\gamma\right) /\left(b^{\alpha}+\gamma\right)}\left(\frac{b^{\alpha}+\gamma}{\alpha\left(1+\ln \left(\left(b^{\alpha}+\gamma\right) / \varepsilon \alpha\right)\right)}\right)^{\left(\left(b^{\alpha}-t^{\alpha}\right) /\left(b^{\alpha}+\gamma\right)\right)},
\end{aligned}
$$

where $\mathbb{D}$ is specified below, $u(x, t)$ is a solution of problems (1)-(3), and $u^{\varepsilon, \gamma}(x, t)$ is a solution of the regularized problem (6). In Section 4, we provide numerical tests to illustrate the theoretical results in the paper.

\section{Statement of the Problem}

Throughout this paper, we denote by $L^{2}(\Omega)$, where $\Omega=[0, a]$, the Hilbert space of Lebesgue measurable functions $v$ on $\Omega$. $\langle\cdot, \cdot\rangle$ and $\|\cdot\|$ represent the inner product and norm on $L^{2}(\Omega)$, respectively. Specifically, the norm and inner product in $L^{2}(\Omega)$ are defined as follows:

$$
\begin{aligned}
\|v\|:=\|v\|_{L^{2}(\Omega)} & =\left(\int_{\Omega} v^{2}(x) \mathrm{d} x\right)^{1 / 2}, \\
\left\langle v_{1}, v_{2}\right\rangle & =\int_{\Omega} v_{1}(x) v_{2}(x) \mathrm{d} x,
\end{aligned}
$$

where $v_{1}, v_{2} \in L^{2}(\Omega)$. Denoting by $C\left([0, b] ; L^{2}(\Omega)\right)$ the space of all continuous functions $v:[0, b] \longrightarrow L^{2}(\Omega)$ and denoting by $|\|\cdot\||$ the sup norm in $C\left([0, b] ; L^{2}(\Omega)\right)$ defined as

$$
|\|v\||:=\|v\|_{C\left([0, b] ; L^{2}(\Omega)\right)}=\sup _{t \in[0, b]}\|v(\cdot, t)\|_{L^{2}(\Omega)} .
$$

The following theorem establishes the formula of the solution to problems (1)-(3). 
Theorem 1. Let $g \in L^{2}(\Omega)$ and let $f: \Omega \times[0, b] \times$ $L^{2}(\Omega) \longrightarrow L^{2}(\Omega)$. Then, the solution of the original problems (1)-(3) has the following form:

$$
u(x, t)=\sum_{n=1}^{\infty}\left(e^{k_{n}\left(\left(b^{\alpha}-t^{\alpha}\right) / \alpha\right)} g_{n}-\int_{t}^{T} s^{\alpha-1} e^{k_{n}\left(\left(s^{\alpha}-t^{\alpha}\right) / \alpha\right)} f_{n}(u)(s) \mathrm{d} s\right) \sin \left(\frac{n \pi}{a} x\right),
$$

where $k_{n}=(n \pi / a)^{2}$ and

$$
\begin{aligned}
u_{n}(t) & =\frac{2}{a}\left\langle u(x, t), \varphi_{n}(x)\right\rangle, \\
g_{n} & =\frac{2}{a}\left\langle g(x), \varphi_{n}(x)\right\rangle, \\
f_{n}(u)(t) & =\frac{2}{a}\left\langle f(x, t, u(x, t)), \varphi_{n}(x)\right\rangle .
\end{aligned}
$$

Proof. By choosing the orthogonal basis $\varphi_{n}(x):=\sin (n \pi x / a), n=1,2, \ldots$, in the Hilbert space $L^{2}(\Omega)$ and by taking the inner product in $L^{2}(\Omega)$ on the two sides of (1), we obtain

$$
\begin{aligned}
D_{t}^{\alpha} & \left\langle u(x, t), \varphi_{n}(x)\right\rangle-\left\langle u_{x x}(x, t), \varphi_{n}(x)\right\rangle \\
& =\left\langle f(x, t, u(x, t)), \varphi_{n}(x)\right\rangle .
\end{aligned}
$$

On the other hand, by using boundary conditions (2), we also get

$$
\begin{aligned}
\left\langle u_{x x}(x, t), \varphi_{n}(x)\right\rangle= & \int_{0}^{a} u_{x x}(x, t) \sin \left(\frac{n \pi}{a} x\right) \mathrm{d} x \\
= & \left.u_{x}(x, t) \sin \left(\frac{n \pi}{a} x\right)\right|_{0} ^{a} \\
& -\left.\frac{n \pi}{a} u(x, t) \cos \left(\frac{n \pi}{a} x\right)\right|_{0} ^{a} \\
& -\left(\frac{n \pi}{a}\right)^{2} \int_{0}^{a} u(x, t) \sin \left(\frac{n \pi}{a} x\right) \mathrm{d} x \\
= & -k_{n}\left\langle u(x, t), \varphi_{n}(x)\right\rangle .
\end{aligned}
$$

Then, it follows from (11)-(13) that

$$
D_{t}^{\alpha} u_{n}(t)+k_{n} u_{n}(t)=f_{n}(u)(t) .
$$

Solving problem (14), we get

$u_{n}(t)=\exp \left(-k_{n} \frac{t^{\alpha}}{\alpha}\right)\left[\int_{0}^{t} s^{\alpha-1} \exp \left(k_{n} \frac{s^{\alpha}}{\alpha}\right) f_{n}(u)(s) \mathrm{d} s+C\right]$.

Basing on (3), we have that

$$
C=g_{n} \exp \left(k_{n} \frac{b^{\alpha}}{\alpha}\right)-\int_{0}^{b} s^{\alpha-1} \exp \left(k_{n} \frac{s^{\alpha}}{\alpha}\right) f_{n}(u)(s) \mathrm{d} s,
$$

where $g_{n}=u_{n}(b)$. Then, (15) yields that

$$
u_{n}(t)=g_{n} \exp \left(k_{n} \frac{b^{\alpha}-t^{\alpha}}{\alpha}\right)-\int_{t}^{b} s^{\alpha-1} \exp \left(k_{n} \frac{s^{\alpha}-t^{\alpha}}{\alpha}\right) f_{n}(u)(s) \mathrm{d} s .
$$

Therefore, the representation of solution of problems (1)-(3) can be written as the infinite series:

$$
\begin{aligned}
u(x, t) & =\sum_{n=1}^{\infty} u_{n}(t) \sin \left(\frac{n \pi}{a} x\right)=\sum_{n=1}^{\infty}\left(g_{n} \exp \left(k_{n} \frac{b^{\alpha}-t^{\alpha}}{\alpha}\right)\right. \\
& \left.-\int_{t}^{b} s^{\alpha-1} \exp \left(k_{n} \frac{s^{\alpha}-t^{\alpha}}{\alpha}\right) f_{n}(u)(s) \mathrm{d} s\right) \sin \left(\frac{n \pi}{a} x\right) .
\end{aligned}
$$

Remark 1. As stated in Section 1, we observe from (18) that when $n$ tends to infinity, the term $\exp \left(k_{n}\left(b^{\alpha}-t^{\alpha}\right) / \alpha\right)$ is increasing rather quickly. Hence, the exact solution given in (18) of problems (1)-(3) is unstable. Thus, problems (1)-(3) are ill posed, and the above term is the unstable factor. So, to regularize the problem or to obtain a stable approximation for problems (1)-(3), we shall replace this unstable factor by a stable one. In this paper, the term $\exp \left(k_{n}\left(b^{\alpha}-t^{\alpha}\right) / \alpha\right)$ is replaced by a stable term which depends on two regularization parameters defined by $\left(\varepsilon k_{n}+e^{-k_{n}\left(\left(b^{\alpha}+\gamma\right) / \alpha\right)}\right)^{\left(\left(t^{\alpha}-b^{\alpha}\right) /\left(b^{\alpha}+\gamma\right)\right)}$, where the first one $(\varepsilon)$ captures the measuring error and the second one $(\gamma)$ captures the regularity of the solution. Therefore, in this paper, we shall use integral equation (6) to approximate or to regularize problems (1)-(3).

\section{Regularization and Error Estimates}

Before investigating the uniqueness and stability of the solution of problem (6), we present the following two inequalities, which will be useful in the proof of the next theorems.

Lemma 1. Let $\alpha \in(0,1)$ be fixed, and $\gamma \geq 0,0<t \leq s \leq b$, $\varepsilon \in \mathscr{D}:=\left(0,\left(b^{\alpha}+\gamma\right) / \alpha\right)$, and $z>0$. Then, the following inequalities hold:

$$
\begin{aligned}
& \left(\varepsilon z+\exp \left(-\frac{\left(b^{\alpha}+\gamma\right)}{\alpha} z\right)\right)^{\left(t^{\alpha}-b^{\alpha}\right) /\left(b^{\alpha}+\gamma\right)} \\
& \leq(\alpha \varepsilon)^{\left(t^{\alpha}-b^{\alpha}\right) /\left(b^{\alpha}+\gamma\right)}\left(\frac{b^{\alpha}+\gamma}{\left(1+\ln \left(b^{\alpha}+\gamma / \alpha \varepsilon\right)\right)}\right)^{\left(\left(b^{\alpha}-t^{\alpha}\right) /\left(b^{\alpha}+\gamma\right)\right)},
\end{aligned}
$$


and

$$
\begin{aligned}
& \exp \left(\left(\frac{s^{\alpha}-b^{\alpha}}{\alpha}\right) z\right)\left(\varepsilon z+\exp \left(-\frac{\left(b^{\alpha}+\gamma\right)}{\alpha} z\right)\right)^{\left(\left(t^{\alpha}-s^{\alpha}\right) /\left(b^{\alpha}+\gamma\right)\right)} \\
& \quad \leq(\alpha \varepsilon)^{\left(\left(t^{\alpha}-s^{\alpha}\right) /\left(b^{\alpha}+\gamma\right)\right)}\left(\frac{b^{\alpha}+\gamma}{\left(1+\ln \left(b^{\alpha}+\gamma / \alpha \varepsilon\right)\right)}\right)^{\left(\left(s^{\alpha}-t^{\alpha}\right) /\left(b^{\alpha}+\gamma\right)\right)} .
\end{aligned}
$$

Proof. Let $b>0, \varepsilon \in \mathscr{D}, \gamma \geq 0$, and $z>0$, and let $\alpha \in(0,1)$ be fixed, then we observe that the function

$$
f(z)=\left(\varepsilon z+\exp \left(-\frac{\left(b^{\alpha}+\gamma\right)}{\alpha} z\right)\right)^{-1}
$$

has the maximum value $z=\ln \left(\left(\left(b^{\alpha}+\gamma\right) / \alpha \varepsilon\right) /\left(\left(b^{\alpha}+\gamma\right) / \alpha\right)\right)$. This yields that

$$
f(z) \leq f\left(\ln \frac{\left(b^{\alpha}+\gamma / \alpha \varepsilon\right)}{\left(b^{\alpha}+\gamma / \alpha\right)}\right)=\frac{b^{\alpha}+\gamma}{\alpha \varepsilon\left(1+\ln \left(\left(b^{\alpha}+\gamma\right) / \alpha \varepsilon\right)\right)} .
$$

Then, we obtain the following inequality:

$$
\left(\varepsilon z+\exp \left(-\frac{\left(b^{\alpha}+\gamma\right)}{\alpha} z\right)\right)^{-\left(\left(b^{\alpha}-t^{\alpha}\right) /\left(b^{\alpha}+\gamma\right)\right)} \leq(\alpha \varepsilon)^{\left(\left(t^{\alpha}-b^{\alpha}\right) /\left(b^{\alpha}+\gamma\right)\right)}\left(\frac{b^{\alpha}+\gamma}{\left(1+\ln \left(\left(b^{\alpha}+\gamma\right) / \alpha \varepsilon\right)\right)}\right)^{\left(\left(b^{\alpha}-t^{\alpha}\right) /\left(b^{\alpha}+\gamma\right)\right)}
$$

Furthermore, we have

$$
\begin{aligned}
\exp \left(\left(\frac{s^{\alpha}-b^{\alpha}}{\alpha}\right) z\right)[f(z)]^{-\left(\left(b^{\alpha}-t^{\alpha}\right) /\left(b^{\alpha}+\gamma\right)\right)} & =\exp \left(\frac{\left(s^{\alpha}-b^{\alpha}\right)}{\alpha\left(b^{\alpha}+\gamma\right)}\left(b^{\alpha}+\gamma\right) z\right)[f(z)]^{\left(\left(s^{\alpha}-b^{\alpha}\right) /\left(b^{\alpha}+\gamma\right)\right)}[f(z)]^{\left(\left(t^{\alpha}-s^{\alpha}\right) /\left(b^{\alpha}+\gamma\right)\right)} \\
& =\left(\varepsilon z \exp \left(\left(\frac{b^{\alpha}+\gamma}{\alpha}\right) z\right)+1\right)^{\left(\left(s^{\alpha}-b^{\alpha}\right) /\left(b^{\alpha}+\gamma\right)\right)}[f(z)]^{\left(\left(t^{\alpha}-s^{\alpha}\right) /\left(b^{\alpha}+\gamma\right)\right)} \\
& \leq[f(z)]^{\left(\left(t^{\alpha}-s^{\alpha}\right) /\left(b^{\alpha}+\gamma\right)\right)}
\end{aligned}
$$

From (23), we obtain

$$
\exp \left(\left(\frac{s^{\alpha}-b^{\alpha}}{\alpha}\right) z\right)[f(z)]^{-\left(\left(b^{\alpha}-t^{\alpha}\right) /\left(b^{\alpha}+\gamma\right)\right)} \leq(\alpha \varepsilon)^{\left(\left(t^{\alpha}-s^{\alpha}\right) /\left(b^{\alpha}+\gamma\right)\right)}\left(\frac{b^{\alpha}+\gamma}{\left(1+\ln \left(b^{\alpha}+\gamma / \alpha \varepsilon\right)\right)}\right)^{\left(\left(s^{\alpha}-t^{\alpha}\right) /\left(b^{\alpha}+\gamma\right)\right)} .
$$

This yields estimate (20).

3.1. The Well Posedness of Regularized Problem (6). In the following theorem, we show that regularized problem (6) is well posed in the sense of Hadamard, i.e., problem (6) has a unique solution, and this solution continuously depends on the given data.

Theorem 2. Let $g \in L^{2}(\Omega)$, and let $f: \Omega \times$ $[0, b] \times L^{2}(\Omega) \longrightarrow L^{2}(\Omega)$ satisfy the globally Lipschitz property with respect to the third variable, i.e., there exists a constant $k>0$ independent of $x, t, v_{1}, v_{2}$ such that

$\left\|f\left(x, t, v_{1}\right)-f\left(x, t, v_{2}\right)\right\| \leq k\left\|v_{1}-v_{2}\right\|, \quad$ for all $v_{1}, v_{2} \in L^{2}(\Omega)$, where $\|\cdot\|$ is the norm in $L^{2}(\Omega)$. Then, regularized problem (6) is well posed in the sense of Hadamard provided that $\alpha \in((1 / 2), 1)$.

Proof. To prove the theorem, we shall divide the proof into two steps. The first step shows that regularized problem (6) has a unique solution $u^{\varepsilon, \gamma} \in C\left([0, b] ; L^{2}(\Omega)\right)$ provided that $\alpha \in((1 / 2), 1)$. In the second step, the continuous dependence of the solution on the data $g$ will be verified.

Step 1. Consider the operator $\mathbb{T}: C([0, b]$; $\left.L^{2}(\Omega)\right) \longrightarrow C\left([0, b] ; L^{2}(\Omega)\right)$ given by

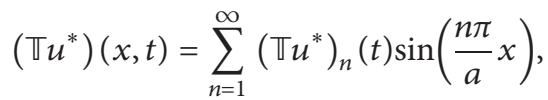

where 


$$
\begin{aligned}
\left(\mathbb{T} u^{*}\right)_{n}(t)= & \Lambda_{1}\left(\varepsilon, \gamma, t, k_{n}\right) g_{n} \\
& -\int_{t}^{b} \Lambda_{2}\left(\varepsilon, \gamma, s, t, k_{n}\right) s^{\alpha-1} f_{n}\left(u^{*}\right)(s) \mathrm{d} s,
\end{aligned}
$$$$
\Lambda_{1}\left(\varepsilon, \gamma, t, k_{n}\right):=\left(\varepsilon k_{n}+\exp \left(-k_{n} \frac{b^{\alpha}+\gamma}{\alpha}\right)\right)^{\left(\left(t^{\alpha}-b^{\alpha}\right) /\left(b^{\alpha}+\gamma\right)\right)},
$$

$$
\begin{aligned}
& \Lambda_{2}\left(\varepsilon, \gamma, s, t, k_{n}\right) \\
& :=\exp \left(k_{n} \frac{s^{\alpha}-b^{\alpha}}{\alpha}\right)\left(\varepsilon k_{n}+\exp \left(-k_{n} \frac{b^{\alpha}+\gamma}{\alpha}\right)\right)^{\left(\left(t^{\alpha}-b^{\alpha}\right) /\left(b^{\alpha}+\gamma\right)\right)} .
\end{aligned}
$$

$$
\lambda(\varepsilon, \gamma)=\left(\frac{b^{\alpha}+\gamma}{\alpha\left(1+\ln \left(b^{\alpha}+\gamma / \alpha \varepsilon\right)\right)}\right) .
$$

It follows from Lemma 1 that

$$
\begin{aligned}
& \Lambda_{1}\left(\varepsilon, \gamma, t, k_{n}\right) \leq\left(\frac{\lambda(\varepsilon, \gamma)}{\varepsilon}\right)^{\left(\left(b^{\alpha}-t^{\alpha}\right) /\left(b^{\alpha}+\gamma\right)\right)}, \\
& \Lambda_{2}\left(\varepsilon, \gamma, t, k_{n}\right) \leq\left(\frac{\lambda(\varepsilon, \gamma)}{\varepsilon}\right)^{\left(\left(s^{\alpha}-t^{\alpha}\right) /\left(b^{\alpha}+\gamma\right)\right)} .
\end{aligned}
$$

Therefore, by Hölder's inequality, one obtains, for $0<t \leq s \leq b$ and $\alpha \in(1 / 2,1)$,

We set

$$
\begin{aligned}
\left|\left(\mathbb{T} u^{*}\right)_{n}(t)-\left(\mathbb{T} u^{*}\right)_{n}(t)\right|^{2} & =\left|\int_{t}^{b} \Lambda_{2}\left(\varepsilon, \gamma, s, t, k_{n}\right) s^{\alpha-1}\left(f_{n}\left(u^{*}\right)(s)-f_{n}\left(v^{*}\right)(s)\right) \mathrm{d} s\right|^{2} \\
& \leq \frac{\lambda^{2}(\varepsilon, \gamma)}{\varepsilon^{2}} C(t)\left(\int_{t}^{b}\left[f_{n}\left(u^{*}\right)(s)-f_{n}\left(v^{*}\right)(s)\right]^{2} \mathrm{~d} s\right),
\end{aligned}
$$

where $\alpha \in(0,1)$ is fixed, $\left(s^{\alpha}-t^{\alpha}\right) /\left(b^{\alpha}+\gamma\right) \in(0,1)$, and $C(t):=\left(\left(b^{2 \alpha-1}-t^{2 \alpha-1}\right) /(2 \alpha-1)\right)$. So, from Lipschitz condition (26), one has that

$$
\begin{aligned}
\left\|\left(\mathbb{T} u^{*}\right)(\cdot, t)-\left(\mathbb{T} u^{*}\right)(\cdot, t)\right\|^{2} & =\frac{a}{2} \sum_{n=1}^{\infty}\left|\left(\mathbb{T} u^{*}\right)_{n}(t)-\left(\mathbb{\mathbb { V }} v^{*}\right)_{n}(t)\right|^{2} \\
& \leq \frac{a}{2} \frac{\lambda^{2}(\varepsilon, \gamma)}{\varepsilon^{2}} C(t) \int_{t}^{b} \sum_{n=1}^{\infty}\left[f_{n}\left(u^{*}\right)(s)-f_{n}\left(v^{*}\right)(s)\right]^{2} \mathrm{~d} s \\
& =\frac{\lambda^{2}(\varepsilon, \gamma)}{\varepsilon^{2}} C(t) \\
& \leq C(t) k^{2} \frac{\lambda^{2}(\varepsilon, \gamma)}{\varepsilon^{2}}(b-t)\left\|u^{*}-v^{*}\right\|^{2} .
\end{aligned}
$$

We observe that the function $C(t)$ is decreasing on $(0, b]$. Hence, $C(t)<C(0)$ for all $t \in(0, b]$. With the same calculation, one also gets

$$
\begin{aligned}
& \left\|\left(\mathbb{T}^{2} u^{*}\right)(\cdot, t)-\left(\mathbb{T}^{2} v^{*}\right)(\cdot, t)\right\|^{2} \\
& \leq \frac{\lambda^{4}(\varepsilon, \gamma)}{\varepsilon^{4}} C^{2}(0) k^{4} \frac{(b-t)^{2}}{2}\left\|u^{*}-v^{*}\right\|^{2},
\end{aligned}
$$

where for $m \geq 1$,

$$
\left(\mathbb{T}^{m} u^{*}\right)_{n}(t)=\Lambda_{1} g_{n}-\int_{t}^{b} \Lambda_{2} s^{\alpha-1} f_{n}\left(\mathbb{T}^{m-1} u^{*}\right)(s) \mathrm{d} s .
$$

Using the mathematical induction method, for $m \geq 3$, one obtains 


$$
\begin{aligned}
& \left\|\left(\mathbb{T}^{m} u^{*}\right)(\cdot, t)-\left(\mathbb{T}^{m} v^{*}\right)(\cdot, t)\right\|^{2} \\
& \leq \frac{\lambda^{2 m}(\varepsilon, \gamma)}{\varepsilon^{2 m}} C^{m}(0) k^{2 m} \frac{(b-t)^{m}}{m !}\left\|u^{*}-v^{*} \mid\right\|^{2} .
\end{aligned}
$$

Now, if we assume that (38) holds for $m=i$, then by Hölder's inequality and from Lemma 1 , for $m=i+1$, we have that

$$
\begin{aligned}
& \left|\left(\mathbb{T}^{i+1} u^{*}\right)_{n}(t)-\left(\mathbb{T}^{i+1} v^{*}\right)_{n}(t)\right|^{2} \\
& \quad \leq \frac{\lambda^{2}(\varepsilon, \gamma)}{\varepsilon^{2}} C(t) \int_{t}^{b}\left[f_{n}\left(\mathbb{T}^{i} u^{*}\right)(s)-f_{n}\left(\mathbb{T}^{i} v^{*}\right)(s)\right]^{2} \mathrm{~d} s .
\end{aligned}
$$

Then, by (38) and Lipschitz condition (26), we have that

$$
\begin{aligned}
\left\|\left(\mathbb{T}^{i+1} u^{*}\right)(\cdot, t)-\left(\mathbb{T}^{i+1} v^{*}\right)(\cdot, t)\right\|^{2} \\
=\frac{a}{2} \sum_{n=1}^{\infty}\left|\left(\mathbb{T}^{i+1} u^{*}\right)_{n}(t)-\left(\mathbb{T}^{i+1} v^{*}\right)_{n}(t)\right|^{2} \\
\leq \frac{a}{2} \frac{\lambda^{2}(\varepsilon, \gamma)}{\varepsilon^{2}} C(t) \int_{t}^{b} \sum_{n=1}^{\infty}\left[f_{n}\left(\mathbb{T}^{i} u^{*}\right)(s)-f_{n}\left(\mathbb{T}^{i} v^{*}\right)(s)\right]^{2} \mathrm{~d} s \\
=\frac{\lambda^{2}(\varepsilon, \gamma)}{\varepsilon^{2}} C(t) \int_{t}^{b}\left\|f\left(\mathbb{T}^{i} u^{*}\right)(\cdot, s)-f\left(\mathbb{T}^{i} v^{*}\right)(\cdot, s)\right\|^{2} \mathrm{~d} s \\
\leq \frac{\lambda^{2}(\varepsilon, \gamma)}{\varepsilon^{2}} C(0) k^{2} \frac{C^{i}(0)\left(\left(a^{2 i}(\varepsilon, \gamma)\right) / \varepsilon^{2 i}\right) k^{2 i}(b-t)^{i+1}}{(i+1) !}\left\|u^{*}-v^{*} \mid\right\|^{2} \\
=\frac{\lambda^{2 i+2}(\varepsilon, \gamma)}{\varepsilon^{2 i+2}} C^{i+1}(0) k^{2 i+2} \frac{(b-t)^{i+1}}{(i+1) !}\left\|u^{*}-v^{*} \mid\right\|^{2},
\end{aligned}
$$

which is inequality (38) for $m=i+1$. Thus, (38) is satisfied for all $m \geq 1$. In addition, inequality (38) is similar to the estimate as follows:

$$
\left\|\left(\mathbb{T}^{m} u^{*}\right)-\left(\mathbb{T}^{m} v^{*}\right)\right\| \leq k^{m} \sqrt{C^{m}(0)} \frac{\lambda^{m}(\varepsilon, \gamma)}{\varepsilon^{m}} \frac{b^{m / 2}}{\sqrt{m !}}\left\|\left|u^{*}-v^{*}\right|\right\|,
$$

where $\lambda(\varepsilon, \gamma)$ is given by (31). Moreover, we have

$$
\lim _{m \rightarrow \infty}\left(\frac{b^{\alpha}+\gamma}{\varepsilon \alpha\left(1+\left(\ln \left(b^{\alpha}+\gamma\right) / \varepsilon \alpha\right)\right)}\right)^{m} \frac{k^{m} b^{m / 2} \sqrt{C^{m}(0)}}{\sqrt{m !}}=0,
$$

where $b>0$ is given and $\alpha \in(1 / 2,1)$ is fixed. Therefore, there exists $m_{0} \in \mathbb{N}$ such that $\mathbb{T}^{m_{0}}$ is a contraction. Thus, problem (6) has a unique solution.

Step 2. Let $v^{\varepsilon, \gamma}$ be a solution of (6) with the data $g$ and $w^{\varepsilon, \gamma}$ be a solution of (6) with the data $h$. From problem (6), we set

$$
\begin{aligned}
& \left(v^{\varepsilon, \gamma}\right)_{n}(t)=\Lambda_{1} g_{n}-\int_{t}^{b} \Lambda_{2} s^{\alpha-1} f_{n}\left(v^{\varepsilon, \gamma}\right)(s) \mathrm{d} s \\
& \left(w^{\varepsilon, \gamma}\right)_{n}(t)=\Lambda_{1} h_{n}-\int_{t}^{b} \Lambda_{2} s^{\alpha-1} f_{n}\left(w^{\varepsilon, \gamma}\right)(s) \mathrm{d} s
\end{aligned}
$$

where $g_{n}=(2 / a)\langle g(x), \sin ((n \pi / a) x)\rangle, \quad h_{n}=(2 / a)\langle h(x)$, and $\sin ((n \pi / a) x)\rangle$. In view of the inequality $\left(z_{1}+z_{2}\right)^{2} \leq$ $2 z_{1}^{2}+2 z_{2}^{2}$, the estimates (32) and (33), and then by Hölder's inequality, we obtain

$$
\begin{aligned}
& \left|\left(v^{\varepsilon, \gamma}\right)_{n}(t)-\left(w^{\varepsilon, \gamma}\right)_{n}(t)\right|^{2} \leq 2\left(\Lambda_{1}\left(h_{n}-g_{n}\right)\right)^{2}+2\left(\int_{t}^{b} \Lambda_{2} s^{\alpha-1}\left(f_{n}\left(w^{\varepsilon, \gamma}\right)(s)-f_{n}\left(v^{\varepsilon, \gamma}\right)(s)\right) \mathrm{d} s\right)^{2} \\
& \quad \leq 2\left(\frac{\lambda(\varepsilon, \gamma)}{\varepsilon}\right)^{2\left(b^{\alpha}-t^{\alpha}\right) /\left(b^{\alpha}+\gamma\right)}\left(g_{n}-h_{n}\right)^{2}+2 C(t) \int_{t}^{b}\left(\frac{\lambda(\varepsilon, \gamma)}{\varepsilon}\right)^{2\left(s^{\alpha}-t^{\alpha}\right) /\left(b^{\alpha}+\gamma\right)}\left(f_{n}\left(w^{\varepsilon, \gamma}\right)(s)-f_{n}\left(v^{\varepsilon, \gamma}\right)(s)\right) \mathrm{d} s .
\end{aligned}
$$

Using Lipschitz condition (26), we have that

$$
\begin{aligned}
& \left\|v^{\varepsilon, \gamma}(\cdot, t)-w^{\varepsilon, \gamma}(\cdot, t)\right\|^{2} \leq 2\left(\frac{\lambda(\varepsilon, \gamma)}{\varepsilon}\right)^{2\left(b^{\alpha}-t^{\alpha}\right) /\left(b^{\alpha}+\gamma\right)}\|g-h\|^{2} \\
& \left.\quad+2\left(\frac{\lambda(\varepsilon, \gamma)}{\varepsilon}\right) \stackrel{\left(\left(-2 t^{\alpha}\right) /\left(b^{\alpha}+\gamma\right)\right)}{\varepsilon}\right)^{2} \int_{t}^{b}\left(\frac{\lambda(\varepsilon, \gamma)}{\varepsilon}\right)^{\left(2 s^{\alpha} /\left(b^{\alpha}+\gamma\right)\right)}\left\|w^{\varepsilon, \gamma}(\cdot, s)-v^{\varepsilon, \gamma}(\cdot, s)\right\|^{2} \mathrm{~d} s .
\end{aligned}
$$



$\underset{\varepsilon)^{\left(2 t^{\alpha} /\left(b^{\alpha}+\gamma\right)\right)}\left\|v^{\varepsilon, \gamma}(\cdot, t)-w^{\varepsilon, \gamma}(\cdot, t)\right\|^{2} \text { and by using Gronwall's }}{\text { Then, }}$ inequality, one gets the following estimate:

$$
\begin{aligned}
& \left\|v^{\varepsilon, \gamma}(\cdot, t)-w^{\varepsilon, \gamma}(\cdot, t)\right\| \\
& \leq \sqrt{2}\left[\frac{b^{\alpha}+\gamma}{\varepsilon \alpha\left(1+\ln \left(\left(b^{\alpha}+\gamma\right) / \varepsilon \alpha\right)\right)}\right]^{\left(\left(b^{\alpha}-t^{\alpha}\right) /\left(b^{\alpha}+\gamma\right)\right)} \\
& \cdot \exp \left(k^{2} C(0)\right)\|g-h\| .
\end{aligned}
$$

This verifies that the solution of (6) depends continuously on the given data. Therefore, the proof is completed.

As stated in Theorem 2, problem (6) is well posed with the condition $\alpha \in(1 / 2,1)$. In the following theorem, we shall extend the restriction of $\alpha$ from the subinterval $(1 / 2,1) \subset(0,1)$ to the interval $\alpha \in(0,1)$. To achieve our aim, we replace the global Lipschitz condition in Theorem 2 with a new Lipschitz condition in the next theorem.
Theorem 3. Let $g \in L^{2}(\Omega)$, and let $f: \Omega \times[0, b] \times L^{2}(\Omega) \longrightarrow L^{2}(\Omega)$ satisfy the following Lipschitz condition:

$\left\|f\left(x, t, v_{1}\right)-f\left(x, t, v_{2}\right)\right\| \leq k t^{\eta}\left\|v_{1}-v_{2}\right\|, \quad \forall v_{1}, v_{2} \in L^{2}(\Omega)$,

where $\alpha \in(0,1)$ is fixed, $\eta \geq(1-\alpha), k>0$ is a constant, and $(x, t) \in \Omega \times(0, b]$. Then, regularized problem (27) is well posed in the sense of Hadamard.

Proof. We follow the ideas in the proof of Theorem 2. We reconsider the operator $\mathbb{T}: C\left([0, b] ; L^{2}(\Omega)\right) \longrightarrow$ $C\left([0, b] ; L^{2}(\Omega)\right)$ given by (27). By Hölder's inequality, Lipschitz condition (47), and from (28), we have that, for $0<t \leq s \leq b$ and $\alpha \in(0,1)$,

$$
\begin{aligned}
\left\|\left(\mathbb{T} u^{*}\right)(\cdot, t)-\left(\mathbb{T} u^{*}\right)(\cdot, t)\right\|^{2} & \leq \frac{a}{2} \frac{\lambda^{2}(\varepsilon, \gamma)}{\varepsilon^{2}}(b-t) \int_{t}^{b} s^{2 \alpha-2} \sum_{n=1}^{\infty}\left[f_{n}\left(u^{*}\right)(s)-f_{n}\left(v^{*}\right)(s)\right]^{2} \mathrm{~d} s \\
& =\frac{\lambda^{2}(\varepsilon, \gamma)}{\varepsilon^{2}}(b-t) \int_{t}^{b} s^{2 \alpha-2}\left\|f\left(u^{*}\right)(\cdot, s)-f\left(v^{*}\right)(\cdot, s)\right\|^{2} \mathrm{~d} s \\
& \leq \frac{\lambda^{2}(\varepsilon, \gamma)}{\varepsilon^{2}} k^{2} b \int_{t}^{b} s^{2(\alpha-1+\gamma)}\left\|u^{*}(\cdot, s)-v^{*}(\cdot, s)\right\|^{2} \mathrm{~d} s \\
& \leq D k^{2} b \frac{\lambda^{2}(\varepsilon, \gamma)}{\varepsilon^{2}}(b-t)\left\|u^{*}-v^{*}\right\|^{2}
\end{aligned}
$$

where $\alpha \in(0,1)$ is fixed, $\eta>1-\alpha$, and $D:=b^{2(\alpha-1+\eta)}$. With the same calculation and using the mathematical induction method, one also gets

$$
\begin{aligned}
& \left\|\left(\mathbb{T}^{m} u^{*}\right)(\cdot, t)-\left(\mathbb{T}^{m} v^{*}\right)(\cdot, t)\right\|^{2} \\
& \leq \frac{\lambda^{2 m}(\varepsilon, \gamma)}{\varepsilon^{2 m}} D^{m} k^{2 m} b^{m} \frac{(b-t)^{m}}{m !}\left\|u^{*}-v^{*}\right\| \|^{2} .
\end{aligned}
$$

Indeed, if we assume that (49) holds for $m=i$, then by using Lemma 1, the Hölder's inequality, and Lipschitz condition (47), for $m=i+1$, we have that 


$$
\begin{aligned}
\left\|\left(\mathbb{T}^{i+1} u^{*}\right)(\cdot, t)-\left(\mathbb{T}^{i+1} v^{*}\right)(\cdot, t)\right\|^{2} & =\frac{a}{2} \sum_{n=1}^{\infty}\left|\left(\mathbb{T}^{i+1} u^{*}\right)_{n}(t)-\left(\mathbb{T}^{i+1} v^{*}\right)_{n}(t)\right|^{2} \\
& \leq \frac{a}{2} \frac{\lambda^{2}(\varepsilon, \gamma)}{\varepsilon^{2}}(b-t) \int_{t}^{b} s^{2(\alpha-1)} \sum_{n=1}^{\infty}\left[f_{n}\left(\mathbb{T}^{i} u^{*}\right)(s)-f_{n}\left(\mathbb{T}^{i} v^{*}\right)(s)\right]^{2} \mathrm{~d} s \\
& =\frac{\lambda^{2}(\varepsilon, \gamma)}{\varepsilon^{2}}(b-t) \int_{t}^{b} s^{2(\alpha-1)}\left\|f\left(\mathbb{T}^{i} u^{*}\right)(\cdot, s)-f\left(\mathbb{T}^{i} v^{*}\right)(\cdot, s)\right\|^{2} \mathrm{~d} s \\
& \leq \frac{\lambda^{2}(\varepsilon, \gamma)}{\varepsilon^{2}} k^{2} b \frac{D^{i+1}\left(a^{2 i}(\varepsilon, \gamma) / \varepsilon^{2 i}\right) k^{2 i} b^{i}(b-t)^{i+1}}{(i+1) !}\left\|u^{*}-v^{*} \mid\right\|^{2} \\
& =\frac{\lambda^{2 i+2}(\varepsilon, \gamma)}{\varepsilon^{2 i+2}} D^{i+1} k^{2 i+2} b^{i+1} \frac{(b-t)^{i+1}}{(i+1) !}\left\|u^{*}-v^{*} \mid\right\|^{2}
\end{aligned}
$$

which is inequality (49) for $m=i+1$. Thus, inequality (49) is satisfied for all $m \geq 1$. Furthermore, inequality (49) yields that

$$
\begin{aligned}
& \left\|\left|\left(\mathbb{T}^{m} u^{*}\right)-\left(\mathbb{T}^{m} v^{*}\right)\right|\right\| \\
& \quad \leq k^{m} \sqrt{D^{m}}\left(\frac{b^{\alpha}+\gamma}{\varepsilon \alpha\left(1+\ln \left(\left(b^{\alpha}+\gamma\right) / \varepsilon \alpha\right)\right)}\right)^{m} \frac{b^{m}}{\sqrt{m !}}\left\|\left|u^{*}-v^{*}\right|\right\| .
\end{aligned}
$$

Hence,

$$
\lim _{m \rightarrow \infty}\left(\frac{b^{\alpha}+\gamma}{\varepsilon \alpha\left(1+\ln \left(\left(b^{\alpha}+\gamma\right) / \varepsilon \alpha\right)\right)}\right)^{m} \frac{k^{m} b^{m} \sqrt{D^{m}}}{\sqrt{m !}}=0
$$

where $b>0$ is given and $\alpha \in(0,1)$ is fixed. So, there exists $m_{0} \in \mathbb{N}$ such that $\mathbb{T}^{m_{0}}$ is a contraction. Thus, problem (6) has a unique solution $u^{\varepsilon, \gamma} \in C\left([0, b] ; L^{2}(\Omega)\right)$ in the case of $\alpha \in(0,1)$. Finally, in order to show that the solution of problem (6) depends continuously on $g$, we assume that $v^{\varepsilon, \gamma}$ and $w^{\varepsilon, \gamma}$ are two solutions of problem (6) corresponding to the final values $g$ and $h$, respectively. Similar to Step 2 in the proof of Theorem 2, by using Lipschitz condition (5), we also obtain

$$
\begin{aligned}
\left\|v^{\varepsilon, \gamma}(\cdot, t)-w^{\varepsilon, \gamma}(\cdot, t)\right\|^{2} \leq & 2\left(\frac{\lambda(\varepsilon, \gamma)}{\varepsilon}\right)^{\left(2\left(b^{\alpha}-t^{\alpha}\right)\right) /\left(b^{\alpha}+\gamma\right)}\|g-h\|^{2} \\
& +2\left(\frac{\lambda(\varepsilon, \gamma)}{\varepsilon}\right)^{\left(-2 t^{\alpha}\right) /\left(b^{\alpha}+\gamma\right)} k^{2} b \int_{t}^{b} s^{2(\alpha-1+\eta)}\left(\frac{\lambda(\varepsilon, \gamma)}{\varepsilon}\right)^{\left(2 s^{\alpha}\right) /\left(b^{\alpha}+\gamma\right)}\left\|w^{\varepsilon, \gamma}(\cdot, s)-v^{\varepsilon, \gamma}(\cdot, s)\right\|^{2} \mathrm{~d} s
\end{aligned}
$$

By putting $\quad H(t)=(\lambda(\varepsilon, \gamma) / \varepsilon)^{\left(2 t^{\alpha} /\left(b^{\alpha}+\gamma\right)\right)}$ $\left\|v^{\varepsilon, \gamma}(\cdot, t)-w^{\varepsilon, \gamma}(\cdot, t)\right\|^{2}$ and $l(t)=s^{2(\alpha-1+\eta)}$ and by Gronwall's inequality, we get

$$
H(t) \leq 2\left(\frac{\lambda(\varepsilon, \gamma)}{\varepsilon}\right)^{\left(2 b^{\alpha} /\left(b^{\alpha}+\gamma\right)\right)} \exp \left(2 k^{2} b \frac{b^{2 \alpha+2 \eta-1}-t^{2 \alpha+2 \eta-1}}{2 \alpha+2 \eta-1}\right)\|g-h\|^{2}
$$

This yields

$$
\left\|v^{\varepsilon, \gamma}(\cdot, t)-w^{\varepsilon, \gamma}(\cdot, t)\right\| \leq \sqrt{2}\left[\frac{b^{\alpha}+\gamma}{\varepsilon \alpha\left(1+\ln \left(\left(b^{\alpha}+\gamma\right) / \varepsilon \alpha\right)\right)}\right]^{\left(b^{\alpha}-t^{\alpha}\right) /\left(b^{\alpha}+\gamma\right)} \cdot \exp \left(k^{2} b \frac{b^{2 \alpha+2 \eta-1}-t^{2 \alpha+2 \eta-1}}{2 \alpha+2 \eta-1}\right)\|g-h\|
$$

Therefore, we can conclude that the solution of (6) depends continuously on the data $g$. The proof is completed.
3.2. Error Estimates. The following theorem presents the error estimate in the case of exact data between the solution $u$ 
of (1)-(3) with the conditional stable and the regularized solution $\mathcal{u}^{\varepsilon, \gamma}$ of (6) without the conditional stable.

Theorem 4. Let $\varepsilon \in \mathscr{D}$ be given, and $f$ and $g$ satisfy the conditions of Theorem 3. Suppose that the unique solution $u$ of
(1)-(3) satisfies the conditional stable $C(\gamma, t, b):=\sum$ ${ }_{n=1}^{\infty} k_{n}^{2} \exp \left(2 k_{n}\left(\left(b^{\alpha}+\gamma\right) / \alpha\right)\right)\left|u_{n}(t)\right|^{2}<\infty, \forall t \in[0, b]$, then we obtain the following estimate:

$$
\left.\left\|u^{\varepsilon, \gamma}(\cdot, t)-u(\cdot, t)\right\| \leq[\lambda(\varepsilon, \gamma)]\right]^{\left(\left(b^{\alpha}-t^{\alpha}\right) /\left(b^{\alpha}+\gamma\right)\right)_{\mathcal{E}}\left(\left(t^{\alpha}+\gamma\right) /\left(b^{\alpha}+\gamma\right)\right)} \sqrt{M} \exp \left(k^{2} b \frac{b^{2 \alpha+2 \eta-1}-t^{2 \alpha+2 \eta-1}}{2 \alpha+2 \eta-1}\right),
$$

where $t \in[0, b], \quad \alpha \in(0,1)$ is fixed, $\quad \eta>(1-\alpha)$, $M=\operatorname{asup}_{t \in[0, b]}\left(\left(\left(b^{\alpha}-t^{\alpha}\right) /\left(b^{\alpha}+\gamma\right)\right)\right)^{2} C(\gamma, t, b)$,

$\lambda(\varepsilon, \gamma):=\left(\left(b^{\alpha}+\gamma\right) / \alpha\left(1+\ln \left(\left(b^{\alpha}+\gamma\right) / \alpha \varepsilon\right)\right)\right)$, and $u^{\varepsilon, \gamma}$ is a unique solution of regularized problem (6).

Proof. Assume that $u$ is a unique solution of (1)-(3), and then based on Theorem 1, one observes that $u$ is represented by

$$
u(x, t)=\sum_{n=1}^{\infty} u_{n}(t) \sin \left(\frac{n \pi}{a} x\right)
$$

where

$$
\begin{aligned}
u_{n}(t)= & \exp \left(k_{n}\left(\frac{b^{\alpha}-t^{\alpha}}{\alpha}\right)\right) g_{n} \\
& -\int_{t}^{b} s^{\alpha-1} \exp \left(k_{n}\left(\frac{s^{\alpha}-t^{\alpha}}{\alpha}\right)\right) f_{n}(u)(s) \mathrm{d} s .
\end{aligned}
$$

Multiplying both sides of (58) with $\left(1+\varepsilon k_{n} \exp \left(k_{n}\left(\left(b^{\alpha}+\gamma\right) / \alpha\right)\right)\right)^{\left(-\left(b^{\alpha}-t^{\alpha}\right)\right) /\left(b^{\alpha}+\gamma\right)}$, one gets

$$
\begin{aligned}
\left(1+\varepsilon k_{n} e^{k_{n}\left(\left(b^{\alpha}-t^{\alpha}\right) / \alpha\right)}\right)^{\left(-\left(b^{\alpha}-t^{\alpha}\right)\right) /\left(b^{\alpha}+\gamma\right)} u_{n}(t)= & e^{k_{n}\left(\left(b^{\alpha}-t^{\alpha}\right) / \alpha\right)}\left(1+\varepsilon k_{n} e^{k_{n}\left(\left(b^{\alpha}+\gamma\right) / \alpha\right)}\right)^{\left(-\left(b^{\alpha}-t^{\alpha}\right)\right) /\left(b^{\alpha}+\gamma\right)} g_{n} \\
& -\int_{t}^{b}\left(1+\varepsilon k_{n} e^{k_{n}\left(\left(b^{\alpha}-t^{\alpha}\right) / \alpha\right)}\right)^{\left(-\left(b^{\alpha}-t^{\alpha}\right)\right) /\left(b^{\alpha}+\gamma\right)} e^{k_{n}\left(\left(b^{\alpha}-t^{\alpha}\right) / \alpha\right)} e^{k_{n}\left(\left(s^{\alpha}-b^{\alpha}\right) / \alpha\right)} s^{\alpha-1} f_{n}(u)(s) \mathrm{d} s \\
= & \left(\varepsilon k_{n}+e^{-k_{n}\left(\left(b^{\alpha}-t^{\alpha}\right) / \alpha\right)}\right)^{\left(\left(t^{\alpha}-b^{\alpha}\right) /\left(b^{\alpha}+\gamma\right)\right)} g_{n} \\
& -\int_{t}^{b}\left(\varepsilon k_{n}+e^{-k_{n}\left(\left(b^{\alpha}-t^{\alpha}\right) / \alpha\right)}\right)^{\left(\left(t^{\alpha}-b^{\alpha}\right) /\left(b^{\alpha}+\gamma\right)\right)} e^{k_{n}\left(\left(s^{\alpha}-b^{\alpha}\right) / \alpha\right)} s^{\alpha-1} f_{n}(u)(s) \mathrm{d} s \\
= & \Lambda_{1} g_{n}-\int_{t}^{b} \Lambda_{2} s^{\alpha-1} f_{n}(u)(s) \mathrm{d} s,
\end{aligned}
$$

where $\Lambda_{1}$ and $\Lambda_{2}$ are denoted as in the proof of Theorem 2 . Moreover, from (6), we have

$$
u_{n}^{\varepsilon, \gamma}(t)=\Lambda_{1} g_{n}-\int_{t}^{b} \Lambda_{2} s^{\alpha-1} f_{n}\left(u^{\varepsilon, \gamma}\right)(s) \mathrm{d} s
$$

Using the inequality $\left(z_{1}+z_{2}\right)^{2} \leq 2 z_{1}^{2}+2 z_{2}^{2}$ and the inequality $1-(1+z)^{-\beta} \leq \beta z$, where $\beta>0$, one has that 


$$
\begin{aligned}
\left|u_{n}(t)-u_{n}^{\varepsilon, \gamma}(t)\right|^{2} \leq & 2\left|u_{n}(t)-\left(1+\varepsilon k_{n} e^{k_{n}\left(\left(b^{\alpha}+\gamma\right) / \alpha\right)}\right)^{\left(-\left(b^{\alpha}-t^{\alpha}\right)\right) /\left(b^{\alpha}+\gamma\right)} u_{n}(t)\right|^{2} \\
& +2\left|u_{n}^{\varepsilon, \gamma}(t)-\left(1+\varepsilon k_{n} e^{k_{n}\left(\left(b^{\alpha}+\gamma\right) / \alpha\right)}\right)^{\left(-\left(b^{\alpha}-t^{\alpha}\right)\right) /\left(b^{\alpha}+\gamma\right)} u_{n}(t)\right|^{2} \\
\leq & 2\left|\int_{t}^{b} \Lambda_{2}\left(\varepsilon, \gamma, s, t, k_{n}\right) s^{\alpha-1}\left[f_{n}\left(u^{\varepsilon, \gamma}\right)(s)-f_{n}(u)(s)\right] \mathrm{d} s\right|^{2} \\
& +2\left|\left(1-\left(1+\varepsilon k_{n} e^{k_{n}\left(\left(b^{\alpha}+\gamma\right) / \alpha\right)}\right)^{\left(-\left(b^{\alpha}-t^{\alpha}\right)\right) /\left(b^{\alpha}+\gamma\right)}\right) u_{n}(t)\right|^{2} \\
\leq & 2\left|\int_{t}^{b} \Lambda_{2}\left(\varepsilon, \gamma, s, t, k_{n}\right) s^{\alpha-1}\left[f_{n}\left(u^{\varepsilon, \gamma}\right)(s)-f_{n}(u)(s)\right] \mathrm{d} s\right|^{2} \\
& +2 k_{n}^{2} \varepsilon^{2}\left(\frac{b^{\alpha}-t^{\alpha}}{b^{\alpha}+\gamma}\right)^{2} e^{2 k_{n}\left(\left(b^{\alpha}+\gamma\right) / \alpha\right)}\left|u_{n}(t)\right|^{2} .
\end{aligned}
$$

Let $C_{n}(\gamma, t, b):=k_{n}^{2} \exp \left(2 k_{n}\left(\left(b^{\alpha}+\gamma\right) / \alpha\right)\right)\left|u_{n}(t)\right|^{2}$. By employing Hölder's inequality and then from Lemma 1 and (61), one obtains

$$
\begin{aligned}
& \left|u_{n}(t)-u_{n}^{\varepsilon, \gamma}(t)\right|^{2} \leq 2(b-t) \int_{t}^{b} \Lambda_{2}^{2}\left(\varepsilon, \gamma, s, t, k_{n}\right) s^{2(\alpha-1)}\left[f_{n}\left(u^{\varepsilon, \gamma}\right)(s)-f_{n}(u)(s)\right]^{2} \mathrm{~d} s+2 \varepsilon^{2}\left(\frac{b^{\alpha}-t^{\alpha}}{b^{\alpha}+\gamma}\right)^{2} C_{n}(\gamma, t, b) \\
& \quad \leq 2(b-t) \int_{t}^{b}\left(\frac{b^{\alpha}+\gamma}{\varepsilon \alpha\left(1+\ln \left(\left(b^{\alpha}+\gamma\right) / \varepsilon \alpha\right)\right)}\right)^{\left(\left(2 s^{\alpha}-2 t^{\alpha}\right) /\left(b^{\alpha}+\gamma\right)\right)} s^{2(\alpha-1)}\left|f_{n}\left(u^{\varepsilon, \gamma}\right)(s)-f_{n}(u)(s)\right|^{2} \mathrm{~d} s+2 \varepsilon^{2}\left(\frac{b^{\alpha}-t^{\alpha}}{b^{\alpha}+\gamma}\right)^{2} C_{n}(\gamma, t, b) .
\end{aligned}
$$

Let

$$
y(z)=z-\varepsilon \alpha-\varepsilon \alpha \ln \left(\frac{z}{\varepsilon \alpha}\right)
$$

for all $z \geq \varepsilon \alpha$. Therefore, for $\varepsilon \in\left(0,\left(b^{\alpha}+\gamma\right) / \alpha\right)$, one gets

$$
\varepsilon \leq \frac{b^{\alpha}+\gamma}{\alpha\left(1+\ln \left(\left(b^{\alpha}+\gamma\right) / \varepsilon \alpha\right)\right)} .
$$

where $\alpha \in(0,1)$ is fixed. It is clear that

$$
y(z) \geq y(\varepsilon \alpha)=\varepsilon \alpha-\varepsilon \alpha-\varepsilon \alpha \ln 1=0,
$$

Hence,

$$
\begin{aligned}
\mathcal{E} & =\varepsilon^{\left(\left(t^{\alpha}+\gamma\right) /\left(b^{\alpha}+\gamma\right)\right)_{\mathcal{E}}\left(\left(b^{\alpha}-t^{\alpha}\right) /\left(b^{\alpha}+\gamma\right)\right)} \leq \varepsilon^{\left(\left(t^{\alpha}+\gamma\right) /\left(b^{\alpha}+\gamma\right)\right)}\left(\frac{b^{\alpha}+\gamma}{\alpha\left(1+\ln \left(\left(b^{\alpha}+\gamma\right) / \varepsilon \alpha\right)\right)}\right)^{\left(\left(b^{\alpha}-t^{\alpha}\right) /\left(b^{\alpha}+\gamma\right)\right)} \\
& =\varepsilon^{\left(\left(t^{\alpha}+\gamma\right) /\left(b^{\alpha}+\gamma\right)\right)}[\lambda(\varepsilon, \gamma)]^{\left(\left(b^{\alpha}-t^{\alpha}\right) /\left(b^{\alpha}+\gamma\right)\right)} .
\end{aligned}
$$

It follows from (62) that 


$$
\begin{aligned}
\left|u_{n}^{\varepsilon, \gamma}(t)-u_{n}(t)\right|^{2} \leq & 2(b-t)[\lambda(\varepsilon, \gamma)]^{\left(2 b^{\alpha}-2 t^{\alpha}\right) /\left(b^{\alpha}+\gamma\right)} \mathcal{E}\left(2\left(t^{\alpha}+\gamma\right)\right) /\left(b^{\alpha}+\gamma\right) \\
& \cdot \int_{t}^{b}[\lambda(\varepsilon, \gamma)]^{\left(2 s^{\alpha}-2 b^{\alpha}\right) /\left(b^{\alpha}+\gamma\right)} \mathcal{E}^{\left(-2\left(s^{\alpha}+\gamma\right)\right) /\left(b^{\alpha}+\gamma\right)} s^{2(\alpha-1)}\left[f_{n}\left(u^{\varepsilon, \gamma}\right)(s)-f_{n}(u)(s)\right]^{2} \mathrm{~d} s \\
& +2[\lambda(\varepsilon, \gamma)]^{\left(2 b^{\alpha}-2 t^{\alpha}\right) /\left(b^{\alpha}+\gamma\right)} \mathcal{E}^{\left(2\left(t^{\alpha}+\gamma\right)\right) /\left(b^{\alpha}+\gamma\right)}\left(\frac{b^{\alpha}-t^{\alpha}}{b^{\alpha}+\gamma}\right)^{2} C_{n}(\gamma, t, b) .
\end{aligned}
$$

Then, by using Lipschitz condition (47), we get the following estimate:

$$
\begin{aligned}
& \left\|u^{\varepsilon, \gamma}(\cdot, t)-u(\cdot, t)\right\|^{2} \leq[\lambda(\varepsilon, \gamma)]^{\left(2 b^{\alpha}-2 t^{\alpha}\right) /\left(b^{\alpha}+\gamma\right)} \mathcal{E}^{\left(2\left(t^{\alpha}+\gamma\right)\right) /\left(b^{\alpha}+\gamma\right)}\left(a \sum_{n=1}^{\infty}\left(\frac{b^{\alpha}-t^{\alpha}}{b^{\alpha}+\gamma}\right)^{2} C_{n}(\gamma, t, b)\right. \\
& \left.+k^{2}(b-t) \int_{t}^{b}[\lambda(\varepsilon, \gamma)]^{\left(2 s^{\alpha}-2 b^{\alpha}\right) /\left(b^{\alpha}+\gamma\right)} \mathcal{E}^{\left(-2\left(s^{\alpha}+\gamma\right)\right) /\left(b^{\alpha}+\gamma\right)} s^{2(\alpha-1+\eta)}\left\|u^{\varepsilon, \gamma}(\cdot, s)-u(\cdot, s)\right\|^{2} \mathrm{~d} s\right) .
\end{aligned}
$$

$\underset{\mathcal{E}^{\left(-2\left(t^{\alpha}+\gamma\right)\right) /\left(b^{\alpha}+\gamma\right)} \underset{\mathrm{Butting}}{\mathrm{\varepsilon}, \gamma}(\cdot, t)-u(\cdot, t) \|^{2}}{H(t)=[\lambda(\varepsilon, \gamma)]^{\left(2 t^{\alpha}-2 b^{\alpha}\right) /\left(b^{\alpha}+\gamma\right)}}$ and $\quad M=$ $\sup _{t \in[0, b]}\left(\left(b^{\alpha}-t^{\alpha}\right) /\left(b^{\alpha}+\gamma\right)\right)^{2} a \sum_{n=1}^{\infty} C_{n}(\gamma, t, b)$, we get the inequality as follows:

$$
H(t) \leq M+2 k^{2} b \int_{t}^{b} s^{2(\alpha-1+\eta)} H(s) \mathrm{d} s .
$$

Using Gronwall's inequality, we have

$$
H(t) \leq M \exp \left(2 k^{2} b \frac{b^{2 \alpha+2 \eta-1}-t^{2 \alpha+2 \eta-1}}{2 \alpha+2 \eta-1}\right) .
$$

This infers that

$$
\left\|u^{\varepsilon, \gamma}(\cdot, t)-u(\cdot, t)\right\| \leq[\lambda(\varepsilon, \gamma)]{ }^{\left(\left(b^{\alpha}-t^{\alpha}\right) /\left(b^{\alpha}+\gamma\right)\right)_{\mathcal{E}}\left(\left(t^{\alpha}+\gamma\right) /\left(b^{\alpha}+\gamma\right)\right)} \sqrt{M} \exp \left(k^{2} b \frac{b^{2 \alpha+2 \eta-1}-t^{2 \alpha+2 \eta-1}}{2 \alpha+2 \eta-1}\right) .
$$

Based on the results of Theorems 3 and 4, the following theorem presents the error estimate in the case of nonexact data between the solution $u$ of (1)-(3) with the conditional stable and regularized solution (6) without the conditional stable.

Theorem 5. Let $\varepsilon \in\left(0,\left(b^{\alpha}+\gamma\right) / \alpha\right)$, where $\gamma$ is a positive parameter, and let $f$ satisfy condition (47). Assume that $u$ is a unique solution of (1)-(3) with the data $g$ which satisfies the conditional stable as in Theorem 4. In addition, let $h$ be the measured data which satisfies

$$
\|g-h\| \leq \varepsilon .
$$

Then, there is a function $v^{\varepsilon, \gamma}$ corresponding to the measured data $h$ such that

$$
\begin{aligned}
& \left\|v^{\varepsilon, \gamma}(\cdot, t)-u(\cdot, t)\right\| \\
& \quad \leq \mathbb{D} \varepsilon^{\left(t^{\alpha}+\gamma\right) /\left(b^{\alpha}+\gamma\right)}\left(\frac{b^{\alpha}+\gamma}{\alpha\left(1+\ln \left(b^{\alpha}+\gamma\right) / \varepsilon \alpha\right)}\right)^{\left(b^{\alpha}-t^{\alpha}\right) /\left(b^{\alpha}+\gamma\right)}, \quad t \in[0, b],
\end{aligned}
$$

where $\mathbb{D}:=(\sqrt{2}+\sqrt{M}) \exp \left(k^{2} b\left(\left(b^{2 \alpha+2 \eta-1}\right)-\left(t^{2 \alpha+2 \eta-1}\right) /\right.\right.$ $(2 \alpha+2 \eta-1)))$ and $M$ is given as in Theorem 4 .

Proof. Denote by $v^{\varepsilon, r}$ and $u^{\varepsilon, \gamma}$ the solutions of (6) with the final values $h$ and $g$, respectively. We observe that

$$
\begin{aligned}
\left\|v^{\varepsilon, r}(\cdot, t)-u(\cdot, t)\right\| \leq & \left\|v^{\varepsilon, r}(\cdot, t)-u^{\varepsilon, r}(\cdot, t)\right\| \\
& +\left\|u^{\varepsilon, r}(\cdot, t)-u(\cdot, t)\right\| .
\end{aligned}
$$

Based on estimates (55) and (56) of Theorems 3 and 4, we have that 


$$
\begin{aligned}
& \left\|v^{\varepsilon, r}(\cdot, t)-u^{\varepsilon, r}(\cdot, t)\right\| \leq \sqrt{2}\left[\frac{\lambda(\varepsilon, \gamma)}{\varepsilon}\right]^{\left(\left(b^{\alpha}-t^{\alpha}\right) /\left(b^{\alpha}+\gamma\right)\right)} \exp \left(k^{2} b \frac{b^{2 \alpha+2 \eta-1}-t^{2 \alpha+2 \eta-1}}{2 \alpha+2 \eta-1}\right)\|g-h\|, \\
& \qquad\left\|u^{\varepsilon, r}(\cdot, t)-u(\cdot, t)\right\| \leq[\lambda(\varepsilon, \gamma)]^{\left(\left(b^{\alpha}-t^{\alpha}\right) /\left(b^{\alpha}+\gamma\right)\right)_{\varepsilon}\left(\left(t^{\alpha}+\gamma\right) /\left(b^{\alpha}+\gamma\right)\right)} \sqrt{M} \exp \left(k^{2} b \frac{b^{2 \alpha+2 \eta-1}-t^{2 \alpha+2 \eta-1}}{2 \alpha+2 \eta-1}\right) . \\
& u(x, b)=\exp \left(\frac{b^{\alpha}}{\alpha}\right) \sin \left(\frac{\pi}{a} x\right), \quad 0 \leq x \leq a .
\end{aligned}
$$

It follows from (72) that, for every $t \in[0, b]$,

$$
\left\|v^{\varepsilon, \gamma}(\cdot, t)-u(\cdot, t)\right\| \leq \mathbb{D} \varepsilon^{\left(\left(t^{\alpha}+\gamma\right) /\left(b^{\alpha}+\gamma\right)\right)}[\lambda(\varepsilon, \gamma)]{ }^{\left(\left(b^{\alpha}-t^{\alpha}\right) /\left(b^{\alpha}+\gamma\right)\right)} \text {, }
$$

where $\mathbb{D}:=(\sqrt{2}+\sqrt{M}) \exp \left(k^{2} b\left(\left(b^{2 \alpha+2 \eta-1}\right)-\left(t^{2 \alpha+2 \eta-1}\right) /\right.\right.$ $(2 \alpha+2 \eta-1)))$. The proof is complete.

\section{Numerical Illustration}

Consider the following linear backward heat problem involving the conformable derivative:

$$
D_{t}^{\alpha} u(x, t)-u_{x x}(x, t)=\left(1+\left(\frac{\pi}{a}\right)^{2}\right) u, \quad 0 \leq x \leq a, 0<t<b
$$

It is not difficult to check that $u(x, t)=\exp \left(t^{\alpha} / \alpha\right) \sin ((\pi / a) x)$ is an exact solution of (77)-(79). It is well known that errors always occur during measurement, and so in this section, we assume the measured data are perturbed by a "noise" with the level $\varepsilon$ as follows:

$$
g^{\text {noise }}(x)=\exp \left(\frac{b^{\alpha}}{\alpha}\right) \sin \left(\frac{\pi}{a} x\right)+\sum_{j=1}^{J_{0}} \varepsilon c_{j} \sin \left(\frac{j \pi}{a} x\right),
$$

where $J_{0} \in \mathbb{N}$ and $c_{j}$ is a sequence of random numbers with mean 0 . Basing on regularized problem (6), we obtain the regularized solution $\mathcal{u}^{\varepsilon, \gamma}$ corresponding to the data $g^{\text {noise }}$ :

$$
u(0, t)=u(a, t)=0, \quad 0<t \leq b
$$

$$
\begin{aligned}
u^{\varepsilon, \gamma}(x, t)= & \left(\varepsilon k_{1}+e^{-k_{1}\left(\left(b^{\alpha}+\gamma\right) / \alpha\right)}\right){ }^{\left(\left(t^{\alpha}-b^{\alpha}\right) /\left(b^{\alpha}+\gamma\right)\right)}\left(e^{b^{\alpha} / \alpha}-\int_{t}^{b} s^{\alpha-1} e^{k_{1}\left(\left(s^{\alpha}-b^{\alpha}\right) / \alpha\right)} f_{1}\left(u^{\varepsilon, \gamma}\right)(s) \mathrm{d} s\right) \sin \left(\frac{\pi}{a} x\right) \\
& +\sum_{j=1}^{J_{0}}\left(\varepsilon k_{j}+e^{-k_{j}\left(\left(b^{\alpha}+\gamma\right) / \alpha\right)}\right)^{\left(\left(t^{\alpha}-b^{\alpha}\right) /\left(b^{\alpha}+\gamma\right)\right)}\left(\varepsilon c_{j}-\int_{t}^{b} s^{\alpha-1} e^{k_{j}} \frac{\left(s^{\alpha}-b^{\alpha}\right)}{\alpha} f_{j}\left(u^{\varepsilon, \gamma}\right)(s) \mathrm{d} s\right) \sin \left(\frac{j \pi}{a} x\right) .
\end{aligned}
$$

Using the concept of the relative error given by

$$
\operatorname{RE}(\varepsilon, t)=\frac{\left\|u^{\varepsilon, \gamma}(\cdot, t)-u(\cdot, t)\right\|}{\|u(\cdot, t)\|},
$$

we shall present the difference between the exact solution and the approximate solution for each $t \in[0, b]$. Now, the following situations are considered:

Situation 1. We focus on the regularization parameter $\varepsilon$. In our numerical example, we take $\gamma=0.3, \alpha=$ $0.8, b=1$, and $a=10$ and let $\varepsilon_{1}=10^{-3}, \varepsilon_{2}=10^{-5}$, and $\varepsilon_{3}=10^{-7}$. The graphs of the solution $u(t)$ and the regularized solution $u^{\varepsilon, \gamma}$ are shown in Figures 1-4 with various values of $\varepsilon$. In addition, we also obtain the error of the proposed method in this paper with random measured data (80) given in Table 1 at $t=0.5$.
Furthermore, let $t=0$, then the graphs of the regularized solution and the exact solution are shown in Figure 5 with the various values of $\varepsilon$.

Remark 2. From Figures 1-4 and Table 1, we observe that the smaller the measurement error $\varepsilon$ is, the closer the regularized solution becomes to the exact solution.

Situation 2. We focus on the parameter $\gamma$. In our numerical example, we take $\varepsilon=10^{-6}$ fixed and let $\alpha=0.5, b=1$, and $a=10$. We consider the values of $\gamma$ : $\gamma_{1}=0$ and $\gamma_{2}=1$. The graphs of the regularized solution in this case are shown in Figures 6 and 7.

Furthermore, let $t=0$, then the graphs of the regularized solution and the exact solution are shown in Figure 8 with the various values of $\gamma$. 


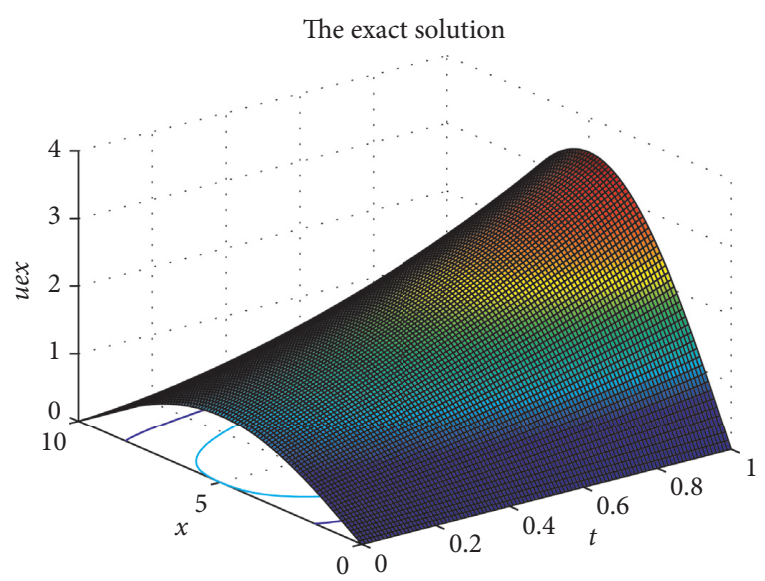

Figure 1: The solution $u$.

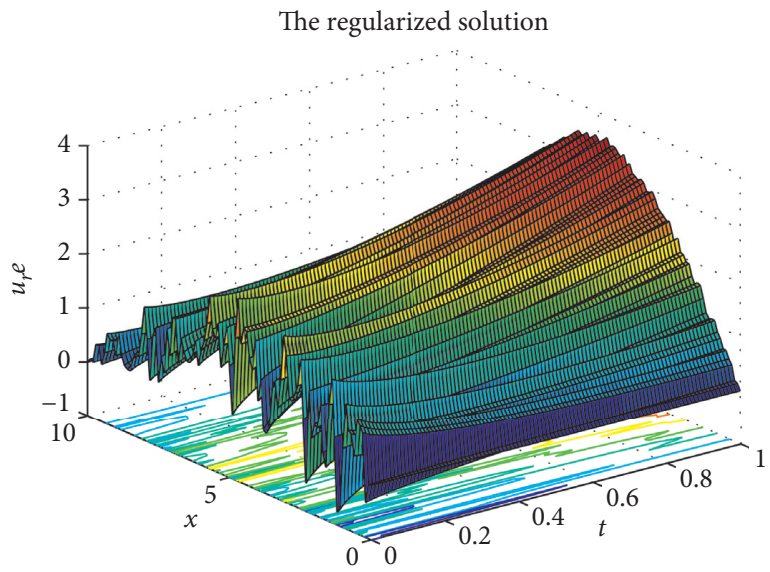

Figure 2: The regularized solution in the case of $\varepsilon_{1}=10^{-3}$.

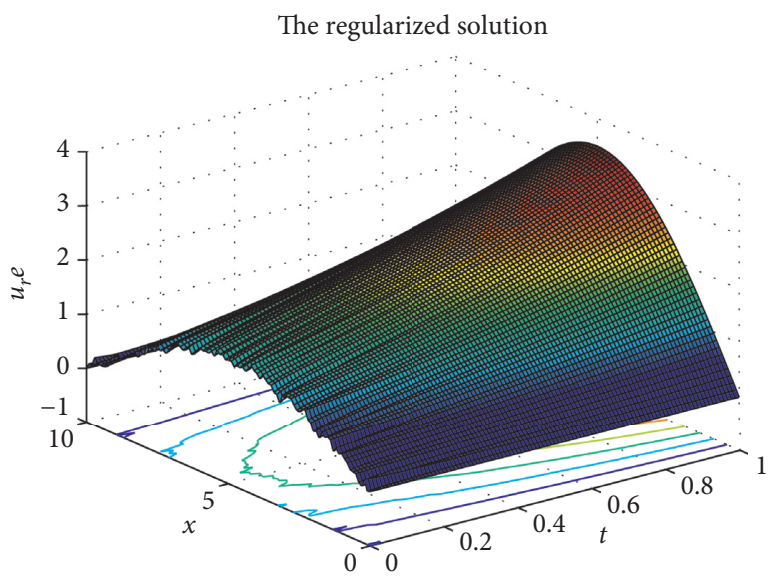

Figure 3: The regularized solution in the case of $\varepsilon_{2}=10^{-5}$.

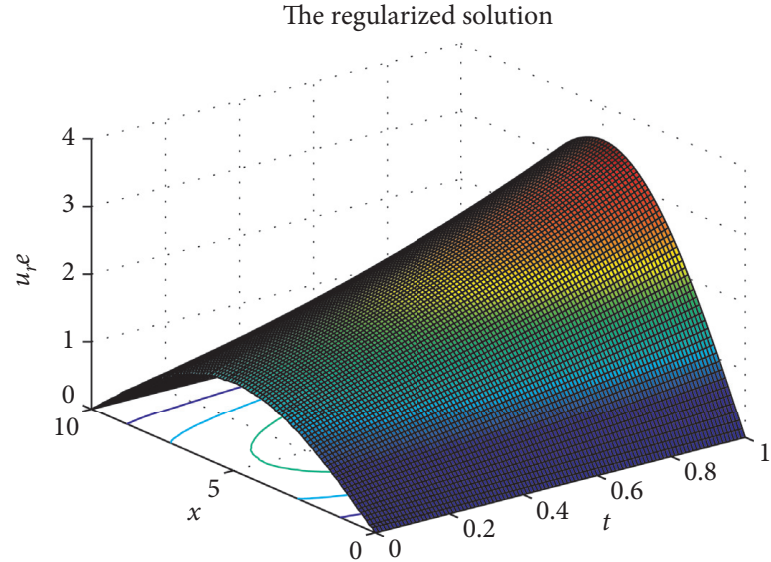

FIgURE 4: The regularized solution in the case of $\varepsilon_{3}=10^{-7}$.

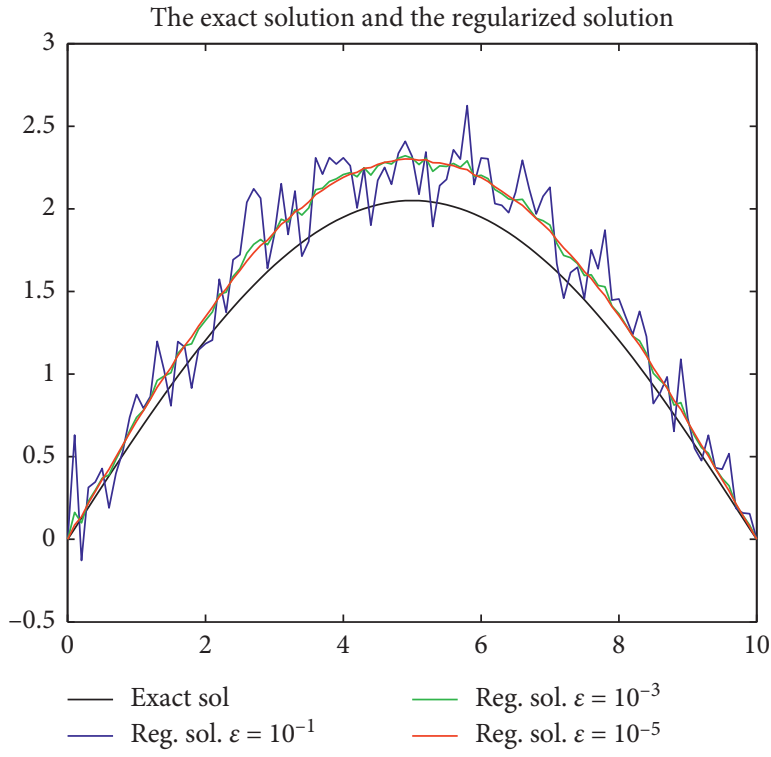

Figure 5: The graphs of $u(t)$ and $u^{\varepsilon, \gamma}(t)$.

TABLE 1: The error of the proposed method.

\begin{tabular}{lcc}
\hline$\varepsilon$ & $\left\|u^{\varepsilon}(\cdot, 0.5)-u(\cdot, 0.5)\right\|$ & $\operatorname{RE}(\varepsilon, 0.5)$ \\
\hline$\varepsilon=10^{-1}$ & 0.748065408 & 5.160107181 \\
$\varepsilon=10^{-2}$ & 0.116350273 & 0.802576715 \\
$\varepsilon=10^{-3}$ & 0.02441038 & 0.16838125 \\
\hline
\end{tabular}

Remark 3. According to Figures 6-8, the regularized solution will be closer to the exact one with a higher value of $\gamma$. It is very useful if we want to obtain a better approximation while the measurement process cannot be improved. 


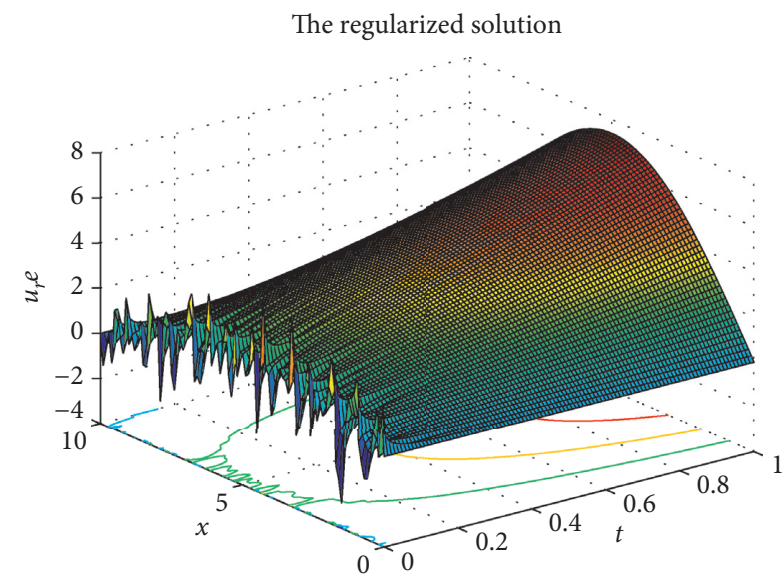

FIGURE 6: The regularized solution $u^{\varepsilon, \gamma_{1}}$ with $\varepsilon=10^{-3}$ and $\gamma_{1}=0$.

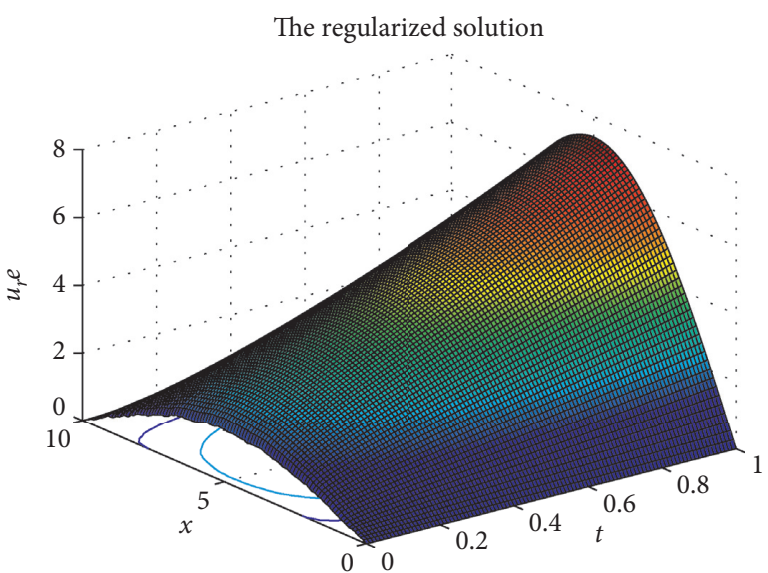

FIGURE 7: The regularized solution $u^{\varepsilon, \gamma_{2}}$ with $\varepsilon=10^{-3}$ and $\gamma_{2}=1$.

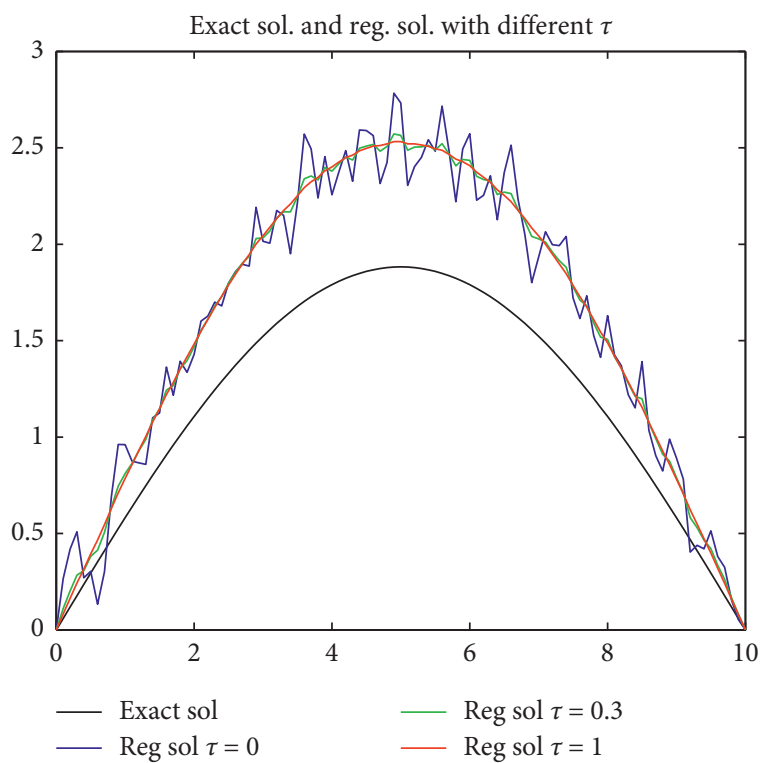

FIGURE 8: The exact solution (black) and the regularized solution with $\varepsilon=10^{-3}$ and $\gamma=0$ (blue), $\gamma=0.3$ (green), and $\gamma=1$ (red).

\section{Conclusion}

In this paper, we have discussed the modified integral equation method involving two regularization parameters for the backward heat problem with a conformable derivative. We have also established error estimates between exact and regularized solutions in the cases of exact data and inexact data. These estimates are supported by several numerical examples.

\section{Data Availability}

No data were used to support this study.

\section{Conflicts of Interest}

The authors declare that they have no conflicts of interest.

\section{Acknowledgments}

The authors would like to thank Dr. Tra Quoc Khanh for his help in doing numerical examples.

\section{References}

[1] A. N. Tikhonov and V. Y. Arsenin, Solutions of Ill-Posed Problems, VH Winston and Sons, Washington, DC, USA, 1977.

[2] V. B. Glasko, Inverse Problems of Mathematical Physics, American Institute of Physics (AIP), New York, NY, USA, 1984.

[3] C.-L. Fu, X.-T. Xiong, and Z. Qian, "Fourier regularization for a backward heat equation," Journal of Mathematical Analysis and Applications, vol. 331, no. 1, pp. 472-480, 2007.

[4] D. N. Hao, N. Van Duc, and D. Lesnic, "Regularization of parabolic equations backward in time by a non-local boundary value problem method," IMA Journal of Applied Mathematics, vol. 75, no. 2, pp. 291-315, 2010.

[5] D. N. Hao and N. V. Duc, "Regularization of backward parabolic equations in Banach spaces," Journal of Inverse and Ill-Posed Problems, vol. 20, no. 5-6, pp. 745-763, 2012.

[6] U. Tautenhahn, "Optimality for ill-posed problems under general source conditions," Numerical Functional Analysis and Optimization, vol. 19, no. 3-4, pp. 377-398, 1998.

[7] D. D. Trong and N. H. Tuan, "Stabilized quasi-reversibility method for a class of nonlinear ill-posed problems," Electronic Journal of Differential Equations, vol. 84, pp. 1-12, 2008.

[8] D. D. Trong and N. H. Tuan, "Regularization and error estimate for the nonlinear backward heat problem using a method of integral equation," Nonlinear Analysis: Theory, Methods and Applications, vol. 71, no. 9, pp. 4167-4176, 2009.

[9] D. D. Trong and T. N. Lien, "Regularization of a discrete backward problem using coefficients of truncated Lagrange polynomials," Electronic Journal of Differential Equations, vol. 51, pp. 1-14, 2007.

[10] N. H. Tuan and H. V. Ngo, "Determination temperature of a backward heat equation with time-dependent coefficients," Mathematica Slovaca, vol. 62, no. 5, pp. 937-948, 2012.

[11] N. V. Hoa and T. Q. Khanh, "Two-parameter regularization method for an axisymmetric inverse heat problem," Boundary Value Problems, vol. 2017, no. 1, pp. 1-12, 2017.

[12] T. Q. Khanh and N. V. Hoa, "On the axisymmetric backward heat equation with non-zero right hand side: regularization 
and error estimates," Journal of Computational and Applied Mathematics, vol. 335, pp. 156-167, 2018.

[13] T. Q. Khanh, T. T. Khieu, and N. V. Hoa, "Stabilizing the nonlinear spherically symmetric backward heat equation via two-parameter regularization method," Journal of Fixed Point Theory and Applications, vol. 19, no. 4, pp. 2461-2481, 2017.

[14] R. Khalil, M. Al Horani, A. Yousef, and M. Sababheh, "A new definition of fractional derivative," Journal of Computational and Applied Mathematics, vol. 264, pp. 65-70, 2014.

[15] A. Atangana, D. Baleanu, and A. Alsaedi, "New properties of conformable derivative," Open Mathematics, vol. 13, pp. 889-898, 2015.

[16] T. Abdeljawad, "On conformable fractional calculus," Journal of Computational and Applied Mathematics, vol. 279, pp. 57-66, 2015.

[17] D. R. Anderson and D. J. Ulness, "Properties of the Katugampola fractional derivative with potential application in quantum mechanics," Journal of Mathematical Physics, vol. 56, no. 6, Article ID 063502, 2015.

[18] I. A. Hammad and R. Khalil, "Fractional Fourier series with applications," American Journal of Computational and Applied Mathematics, vol. 4, pp. 187-191, 2014.

[19] W. S. Chung, "Fractional Newton mechanics with conformable fractional derivative," Journal of Computational and Applied Mathematics, vol. 290, pp. 150-158, 2015.

[20] M. Eslami, "Exact traveling wave solutions to the fractional coupled nonlinear Schrodinger equations," Applied Mathematics and Computation, vol. 285, pp. 141-148, 2016.

[21] Y. Çenesiz, D. Baleanu, A. Kurt, and O. Tasbozan, "New exact solutions of Burgers' type equations with conformable derivative," Waves in Random and Complex Media, vol. 27, pp. 1-14, 2017.

[22] Y. Çenesiz and A. Kurt, "The solutions of time and space conformable fractional heat equations with conformable Fourier transform," Acta Universitatis Sapientiae, Mathematica, vol. 7, no. 2, pp. 130-140, 2015.

[23] Y. Çenesiz, A. Kurt, and E. Nane, "Stochastic solutions of conformable fractional Cauchy problems," Statistics \& Probability Letters, vol. 124, pp. 126-131, 2017.

[24] H. Vu, D. O’Regan, and H. V. Ngo, "Regularization and error estimates for an inverse heat problem under the conformable derivative," Open Mathematics, vol. 16, no. 1, pp. 999-1011, 2018. 